\title{
ORIGINAL
}

\section{ESTIMACIÓN DE LA PREVALENCIA DEL HIPOTIROIDISMO EN CASTILLA Y LEÓN Y SU EVOLUCIÓN DESDE 1992 AL 2000 A TRAVÉS DEL CONSUMO DE HORMONAS TIROIDEAS}

\author{
Alfonso Díaz Madero (1) y Adela López Ferreras (2) \\ (1) Farmacéutico de la Gerencia de Atención Primaria de Zamora \\ (2) Licenciada en Farmacia
}

\section{RESUMEN}

Fundamento: La importancia que tiene el hipotiroidismo desde el punto de vista epidemiológico y la dificultad de disponer de unos datos actualizados, nos ha llevado a realizar este estudio cuyo objetivo es conocer el consumo de hormonas tiroideas en la población general en el ámbito de la seguridad social en la Comunidad de Castilla y León, por provincias, así como su evolución desde 1992 al 2000, como indicador estimativo de la prevalencia del hipotiroidismo.

Métodos: A partir de la base de datos ECOM del Ministerio de Sanidad y Consumo, se obtienen el número de envases de hormonas tiroideas dispensados con receta de la seguridad social, en las provincias de Castilla y León durante el periodo 1992-2000, estimando la prevalencia por medio de las dosis diarias definidas (DDD) por 100.000 habitantes.

Resultados: Se estima una prevalencia de hipotiroidismo en Castilla y León de 185 casos por 100.000 habitantes en 1992 que aumenta a 489 en el 2000, constituyendo un incremento del $164 \%$. Ello representa en 1992 entre 4.559-4.827 enfermos y entre 11.929-12.360 en el 2000. El aumento de la prevalencia se observa en todas las provincias. Las provincias con mayor prevalencia son León, Soria y Ávila, siendo Palencia, Salamanca y Burgos las de menor.

Conclusiones: La especificidad del tratamiento nos ha permitido establecer una relación entre el consumo de hormonas tiroideas y la prevalencia del hipotiroidismo, así como su evolución desde 1992 al 2000, aproximándonos a la situación actual de esta patología en Castilla y León.

Palabras clave: Hipotiroidismo. Farmacoepidemiología. Hormonas tiroides. Dosis diaria definida.

Correspondencia:

Alfonso Díaz Madero

Farmacéutico de la Gerencia de Atención Primaria

C/ Santa Elena s/n. 49007 ZAMORA

Correo electrónico: adiaz@gapza11.insalud.es

\section{ABSTRACT}

Estimate of the Prevalence of Hypothyroidism in Castilla y León and its Progress from 1992 to 2000 through the Consumption of Thyroid Hormones

Background: The significance of hypothyroidism from an epidemiological viewpoint and the difficulty of obtaining updated information have led us to undertake this study, the objective of which is to ascertain the level of consumption of thyroid hormones in the general population within the scope of the Social Security health care system in the Community of Castilla y León, by provinces, together with its progress from 1992 to 2000, as an estimative indicator of the prevalence of hypothyroidism.

Methods: With the ECOM data base of the Ministry of Health and Consumer Affairs as our starting point, we obtained the amounts of thyroid hormones dispensed with prescriptions issued by the Social Security health care services in the provinces of Castilla y León during the period between 1992-2000, estimating the prevalence by means of the Daily Defined Doses (DDD) per 100,000 inhabitants.

Results: The prevalence of hypothyroidism in Casti1la-León is estimated at 185 cases per 100,000 inhabitants in 1992 , increasing to 489 in 2000 , reflecting a percentage rise of $164 \%$. This result represents between 4,559-4,827 patients in 1992 , and between $11,929-12,360$ in 2000 . The increase in prevalence was observed in all of the provinces.

The provinces with the highest prevalence are León, Soria and Avila, while Palencia, Salamanca and Burgos have the least incidence.

Conclusions: The specificity of the treatment has enabled us to establish a relationship between the consumption of thyroid hormones and the prevalence of hypothyroidism, as well as its progress from 1992 to 2000, thus providing us with a close estimate of the current situation of this pathology in Castilla-León.

Key Words: Hypothyroidism, Thyroid Hormones, Defined Daily Dose, Phamocoepidemiologie. 


\section{INTRODUCCIÓN}

El hipotiroidismo es una situación clínica caracterizada por un déficit de secreción de hormonas tiroideas, producida por una alteración orgánica o funcional de la misma glándula (hipotiroidismo primario) o por un déficit de estimulación de la hormona estimulante del tiroides o TSH (hipotiroidismo secundario). Aunque en la mayoría de los casos es una situación crónica e irreversible, existen determinadas patologías que pueden cursar con una hipofunción tiroidea transitoria $^{1}$.

En el hipotiroidismo primario, es útil considerar la existencia o ausencia de bocio lo que delimita bastante sus posibles causas. En las zonas de bocio endémico la causa más frecuente de hipotiroidismo es el déficit de yodo, cuyo exceso, paradójicamente, también puede producirlo. En países occidentales la tiroiditis crónica autoinmune o enfermedad de Hashimoto y los tratamientos destructivos previos de la glándula son las principales causas. El tratamiento con radioyodo o con cirugía es causa de hasta un tercio de los casos de hipotiroidismo. El hipotiroidismo secundario suele aparecer en el contexto de un cuadro de déficit global hipotálamo-hipofisario, de diverso origen ${ }^{1}$. En el $95 \%$ de los casos se trata de un hipotiroidismo primario, es decir, por alteraciones tiroideas. En el resto, la causa del hipotiroidismo está en el hipotálamo, la hipófisis o en la resistencia periférica a las hormonas tiroideas $^{2}$.

El funcionamiento anormal del tiroides tiene múltiples implicaciones en salud pública, ya que se trata de trastornos frecuentes que si no son diagnosticados y tratados pueden empeorar o complicar enfermedades concomitantes como trastornos cardiacos, diabetes, hipertensión, osteoporosis, etc. La magnitud del problema no se conoce completamente ${ }^{3}$, afectando a una proporción considerable de la población, sin embargo la prevalencia depende de factores étnicos y geográficos ${ }^{4}$. La prevalencia del hipotiroi- dismo en la población general es del 1,4\% en las mujeres adultas y del $0,1 \%$ en los hombres. En la población mayor de 65 años, la prevalencia aumenta hasta un $2-4 \%$, siendo de un $6 \%$ en mujeres ${ }^{1}$. En Lérida, Ibarz et $\mathrm{al}^{5}$ obtienen en 1994-95 una prevalencia del hipotiroidismo de $3,48 \pm 1,7 \%$, cifra superior a la obtenida en la Cerdaña por Vila ${ }^{6}$ en 1990 con una prevalencia del 1,21\%.

Estudios realizados en distintos países difieren en la estimación de la prevalencia para el hipotiroidismo: En el estudio longitudinal realizado en Whickham (Reino Unido $)^{7}$, la prevalencia era de $0,8-1,1 \%$ siendo la edad de los pacientes de 30 a 76 años. Bjoro et $\mathrm{al}^{4}$ encontraron en su estudio realizado en Noruega una prevalencia del hipotiroidismo del $0,9 \%$ en varones y del $4,8 \%$ en mujeres. Sin embargo, según un estudio ${ }^{8}$ realizado en una población urbana americana la prevalencia era del $6,9 \%$ en los mayores de 55 años, siendo del 10,3\% en ancianos según el estudio Sawin et $\mathrm{al}^{9}$ realizado en EEUU. Por otro lado, el estudio realizado en Inglaterra por Hunter et $\mathrm{al}^{10}$ pone de manifiesto que la prevalencia del hipotiroidismo es del $0,135 \%$ en personas menores de 22 años y del $0,113 \%$ en el grupo de 11-18 años. De todos estos estudios se concluye que la prevalencia del hipotiroidismo es mayor en mujeres que en hombres y que aumenta con la edad.

La prevalencia de esta enfermedad también puede ser estimada de forma fiable a través del consumo de fármacos específicos, como las hormonas tiroideas, dado que la sintomatología se debe a un bajo nivel de estas hormonas y su administración constituye el tratamiento específico ${ }^{11}$. La estimación de la prevalencia del hipotiroidismo en una comunidad es importante desde el punto de vista epidemiológico, ya que no sólo permitirá conocer si existen diferencias interprovinciales, sino también establecer su situación respecto a otras áreas geográficas y así plantear la necesidad de programas de cribado que permitan detectar casos de hipotiroidismo no diagnosticado ${ }^{5}$. La dificultad de 
disponer de unos datos actualizados, nos ha llevado a realizar este estudio, cuyo objetivo es conocer, como indicador estimativo de la prevalencia del hipotiroidismo, el consumo de hormonas tiroideas en la población general en el ámbito de la seguridad social en la Comunidad de Castilla y León en general y en sus provincias en particular, así como su evolución desde 1992 al 2000.

\section{MATERIAL Y MÉTODOS}

Se realiza un estudio descriptivo retrospectivo de las dispensaciones efectuadas por las oficinas de farmacia de la Comunidad de Castilla y León, con recetas de la Seguridad Social en las que se prescribió alguna hormona tiroidea del grupo terapéutico H03A, en el periodo 1992-2000.

Castilla y León es una Comunidad de 2.543.820 habitantes (censo de 1991), de los cuales 454.701 son mayores de 65 años. Su extensión es de $94.224 \mathrm{Km}^{2}$ de superficie, dividido en 243 zonas básicas de salud y con 14 hospitales con lo que se cubre el derecho a la salud del $94,71 \%$ de sus habitantes ${ }^{12}$.

Como fuente de datos se utilizó la base de datos ECOM del Ministerio de Sanidad y Consumo, que contiene información sobre el número de envases de especialidades farmacéuticas dispensadas con receta de la Seguridad Social por las oficinas de farmacia. El consumo obtenido es considerado una aproximación aceptable del consumo real ${ }^{13}$. La búsqueda se llevó a cabo para cada provincia de la Comunidad, en el periodo 1992-2000 y para los siguientes principios activos: levotiroxina, liotironina, extracto de tiroides y tiroglobulina.

La prevalencia se midió a través del indicador Dosis Diaria Definida (DDD) por 100.000 habitantes, que estima, con carácter de tasa poblacional, el número de personas que en un momento dado (prevalencia de punto) están consumiendo una dosis terapéutica diaria de hormona tiroidea para el tratamiento de hipotiroidismo ${ }^{11}$. La DDD es la dosis media diaria de mantenimiento en adultos de un medicamento cuando se utiliza en su principal indicación ${ }^{13}$.

Para este estudio se han utilizado las DDD establecidas en el Nomenclator Digitalis ${ }^{14}$ por ser el más actualizado y completo, ya que figuran todos los fármacos comercializados en España con sus DDD y el origen $\mathrm{o}$ autor de éstas. Para todas las utilizadas figura como origen la Anatomical Therapeutic Chemical (ATC) classification index, excepto para tiroglobulina que aparece como origen o autor el Insalud. Las DDD utilizadas han sido: Tiroglobulina $85 \mathrm{mg}$, Extracto de tiroides $0,15 \mathrm{mg}$, Liotironina $0,06 \mathrm{mg}$ y Levotiroxina $0,15 \mathrm{mg}$.

Para cada provincia y año se calcula la DDD por 100.000 habitantes mediante la fórmula ${ }^{13,15,16}$ :

$$
\begin{gathered}
{[\Sigma(\text { número de DDD de cada principio }} \\
\text { activo }) \times 100.000 \text { habitantes }] / \\
\text { [población } \times 365 \text { días] }
\end{gathered}
$$

Número de DDD de un principio activo = miligramos de principio activo consumidos en un año dividido por su DDD en miligramos.

Miligramos de principio activo consumidos en un año $=\Sigma$ (n. ${ }^{\circ}$ de envases dispensados anualmente de cada especialidad farmacéutica $\times \mathrm{n} .{ }^{\circ}$ de formas farmacéuticas por envase $\times$ miligramos de principio activo por forma farmacéutica).

La DDD por 100.000 habitantes expresa, en consecuencia, el número de dosis promedio que se han prescrito para el consumo en un día, lo que se equipara a las personas consumidoras de una dosis terapéutica diaria promedio por cada 100.000 habitantes. Ello nos ofrece una tasa de prevalencia de enfermos en un día del período estudiado por 100.000 habitantes, técnica utilizada en otros estudios similares ${ }^{11,17}$. Para cada DDD por 100.000 habitantes obtenido se estima el intervalo de confianza al $95 \%{ }^{18}$. 
Los datos de población se obtienen de las proyecciones y estimaciones intercensales de población de derecho publicadas por el Instituto Nacional de Estadística para los años del periodo de estudio ${ }^{19}$.

Los criterios de evaluación utilizados son:

1. DDD por 100.000 habitantes de hormonas tiroideas y su intervalo de confianza al 95\% para cada provincia de la Comunidad autónoma en el periodo 1992-2000.

2. Porcentaje de incremento en el año 2000 respecto a 1992:

$$
\frac{\left(\mathrm{DDD} \cdot 10^{5}\right)_{2000}-\left(\mathrm{DDD} \cdot 10^{5}\right)_{1992}}{\left(\mathrm{DDD} \cdot 10^{5}\right)_{1992}} \times 100
$$

\section{RESULTADOS}

Considerando las hormonas tiroideas como tratamiento específico de todos los pacientes con hipotiroidismo, se estima una prevalencia en la Comunidad de Castilla y León de 185 casos por 100.000 habitantes en 1992 y de 489 casos por 100.000 habitantes en el año 2000, lo que constituye un incremento del $164 \%$. Ello representa entre 4.559 y 4.827 enfermos en 1992 y entre 11.929 y 12.360 enfermos en el año 2000.

En la tabla 1 se presenta la evolución de la prevalencia expresada en DDD por 100.000 habitantes y su intervalo de confianza al $95 \%$ en las provincias de Castilla y León, así como el incremento en el periodo objeto de estudio. Las provincias que experimentan un mayor incremento en este periodo han sido Burgos, Valladolid y Segovia.

Tabla 1

Evolución de las DDD por 100.000 habitantes en las provincias de Castilla y León desde 1992 al 2000, sus intervalos de confianza al $95 \%$ e incremento en el año 2000 respecto a 1992

\begin{tabular}{|c|c|c|c|c|c|c|c|c|c|c|}
\hline & 1992 & 1993 & 1994 & 1995 & 1996 & 1997 & 1998 & 1999 & 2000 & $\begin{array}{c}\% \text { Incre- } \\
\text { mento } \\
2000 / 1992\end{array}$ \\
\hline ÁVILA & $\begin{array}{c}256 \\
(232-279)\end{array}$ & $\begin{array}{c}282 \\
(257-307)\end{array}$ & $\begin{array}{c}310 \\
(284-337)\end{array}$ & $\begin{array}{c}348 \\
(320-376)\end{array}$ & $\begin{array}{c}398 \\
(368-428)\end{array}$ & $\begin{array}{c}430 \\
(398-461)\end{array}$ & $\begin{array}{c}467 \\
(434-500)\end{array}$ & $\begin{array}{c}508 \\
(474-542)\end{array}$ & $\begin{array}{c}562 \\
(526-598)\end{array}$ & 120 \\
\hline BURGOS & $\begin{array}{c}142 \\
(130-154)\end{array}$ & $\begin{array}{c}151 \\
(138-164)\end{array}$ & $\begin{array}{c}174 \\
(161-188)\end{array}$ & $\begin{array}{c}197 \\
(182-212)\end{array}$ & $\begin{array}{c}243 \\
(227-259)\end{array}$ & $\begin{array}{c}275 \\
(258-293)\end{array}$ & $\begin{array}{c}309 \\
(291-328)\end{array}$ & $\begin{array}{c}366 \\
(346-386)\end{array}$ & $\begin{array}{c}428 \\
(407-450)\end{array}$ & 201 \\
\hline LEÓN & $\begin{array}{c}259 \\
(245-272)\end{array}$ & $\begin{array}{c}275 \\
(260-289)\end{array}$ & $\begin{array}{c}281 \\
(267-296)\end{array}$ & $\begin{array}{c}316 \\
(301-331)\end{array}$ & $\begin{array}{c}379 \\
(362-396)\end{array}$ & $\begin{array}{c}414 \\
(397-432)\end{array}$ & $\begin{array}{c}470 \\
(451-488)\end{array}$ & $\begin{array}{c}538 \\
(518-558)\end{array}$ & $\begin{array}{c}626 \\
(604-648)\end{array}$ & 142 \\
\hline PALENCIA & $\begin{array}{c}140 \\
(123-157)\end{array}$ & $\begin{array}{c}162 \\
(143-180)\end{array}$ & $\begin{array}{c}185 \\
(166-205)\end{array}$ & $\begin{array}{c}206 \\
(185-227)\end{array}$ & $\begin{array}{c}244 \\
(221-266)\end{array}$ & $\begin{array}{c}260 \\
(237-283)\end{array}$ & $\begin{array}{c}279 \\
(255-304)\end{array}$ & $\begin{array}{c}322 \\
(296-349)\end{array}$ & $\begin{array}{c}381 \\
(353-410)\end{array}$ & 173 \\
\hline SALAMANCA & $\begin{array}{c}145 \\
(133-158)\end{array}$ & $\begin{array}{c}162 \\
(149-175)\end{array}$ & $\begin{array}{c}184 \\
(170-198)\end{array}$ & $\begin{array}{c}208 \\
(193-223)\end{array}$ & $\mid \begin{array}{c}243 \\
(226-259)\end{array}$ & $\begin{array}{c}276 \\
(258-293)\end{array}$ & $\begin{array}{c}305 \\
(287-323)\end{array}$ & $\begin{array}{c}341 \\
(322-361)\end{array}$ & $\begin{array}{c}384 \\
(363-404)\end{array}$ & 164 \\
\hline SEGOVIA & $\begin{array}{c}159 \\
(139-179)\end{array}$ & $\begin{array}{c}179 \\
(158-201)\end{array}$ & $\begin{array}{c}201 \\
(178-224)\end{array}$ & $\begin{array}{c}228 \\
(204-253)\end{array}$ & $\begin{array}{c}285 \\
(258-312)\end{array}$ & $\begin{array}{c}340 \\
(310-369)\end{array}$ & $\begin{array}{c}359 \\
(328-389)\end{array}$ & $\begin{array}{c}394 \\
(362-426)\end{array}$ & $\begin{array}{c}462 \\
(427-497)\end{array}$ & 190 \\
\hline SORIA & $\begin{array}{c}243 \\
(212-275)\end{array}$ & $\begin{array}{c}258 \\
(226-291)\end{array}$ & $\begin{array}{c}282 \\
(248-316)\end{array}$ & $\begin{array}{c}298 \\
(263-333)\end{array}$ & $\begin{array}{c}343 \\
(305-381)\end{array}$ & $\begin{array}{c}382 \\
(342-422)\end{array}$ & $\begin{array}{c}414 \\
(373-456)\end{array}$ & $\begin{array}{c}476 \\
(432-521)\end{array}$ & $\begin{array}{c}587 \\
(538-637)\end{array}$ & 141 \\
\hline VALLADOLID & $\begin{array}{c}159 \\
(148-170)\end{array}$ & $\begin{array}{c}184 \\
(172-196)\end{array}$ & $\begin{array}{c}217 \\
(204-230) \\
\end{array}$ & $\begin{array}{c}242 \\
(229-256)\end{array}$ & $\begin{array}{c}292 \\
(277-307)\end{array}$ & $\begin{array}{c}324 \\
(308-340)\end{array}$ & $\begin{array}{c}368 \\
(351-385)\end{array}$ & $\begin{array}{c}416 \\
(398-434)\end{array}$ & $\begin{array}{c}477 \\
(458-497)\end{array}$ & 200 \\
\hline ZAMORA & $\begin{array}{c}175 \\
(158-193)\end{array}$ & $\begin{array}{c}192 \\
(173-210)\end{array}$ & $\begin{array}{c}209 \\
(189-228) \\
\end{array}$ & $\begin{array}{c}234 \\
(213-255) \\
\end{array}$ & $\begin{array}{c}277 \\
(255-300) \\
\end{array}$ & $\begin{array}{c}310 \\
(286-335)\end{array}$ & $\begin{array}{c}355 \\
(329-380) \\
\end{array}$ & $\begin{array}{c}415 \\
(387-443) \\
\end{array}$ & $\begin{array}{c}473 \\
(443-503) \\
\end{array}$ & 170 \\
\hline $\begin{array}{l}\text { CASTILLA Y } \\
\text { LEÓN }\end{array}$ & $\begin{array}{c}185 \\
(180-190)\end{array}$ & $\begin{array}{c}203 \\
(198-209)\end{array}$ & $\begin{array}{c}224 \\
(219-230)\end{array}$ & $\begin{array}{c}251 \\
(245-258)\end{array}$ & $\mid \begin{array}{c}300 \\
(293-307)\end{array}$ & $\begin{array}{c}333 \\
(326-340)\end{array}$ & $\begin{array}{c}372 \\
(365-380)\end{array}$ & $\mid \begin{array}{c}424 \\
(416-432)\end{array}$ & $\begin{array}{c}489 \\
(481-498)\end{array}$ & 164 \\
\hline
\end{tabular}


Figura 1

Evolución desde 1992 al 2000 de la prevalencia del hipotiroidismo en Castilla y León

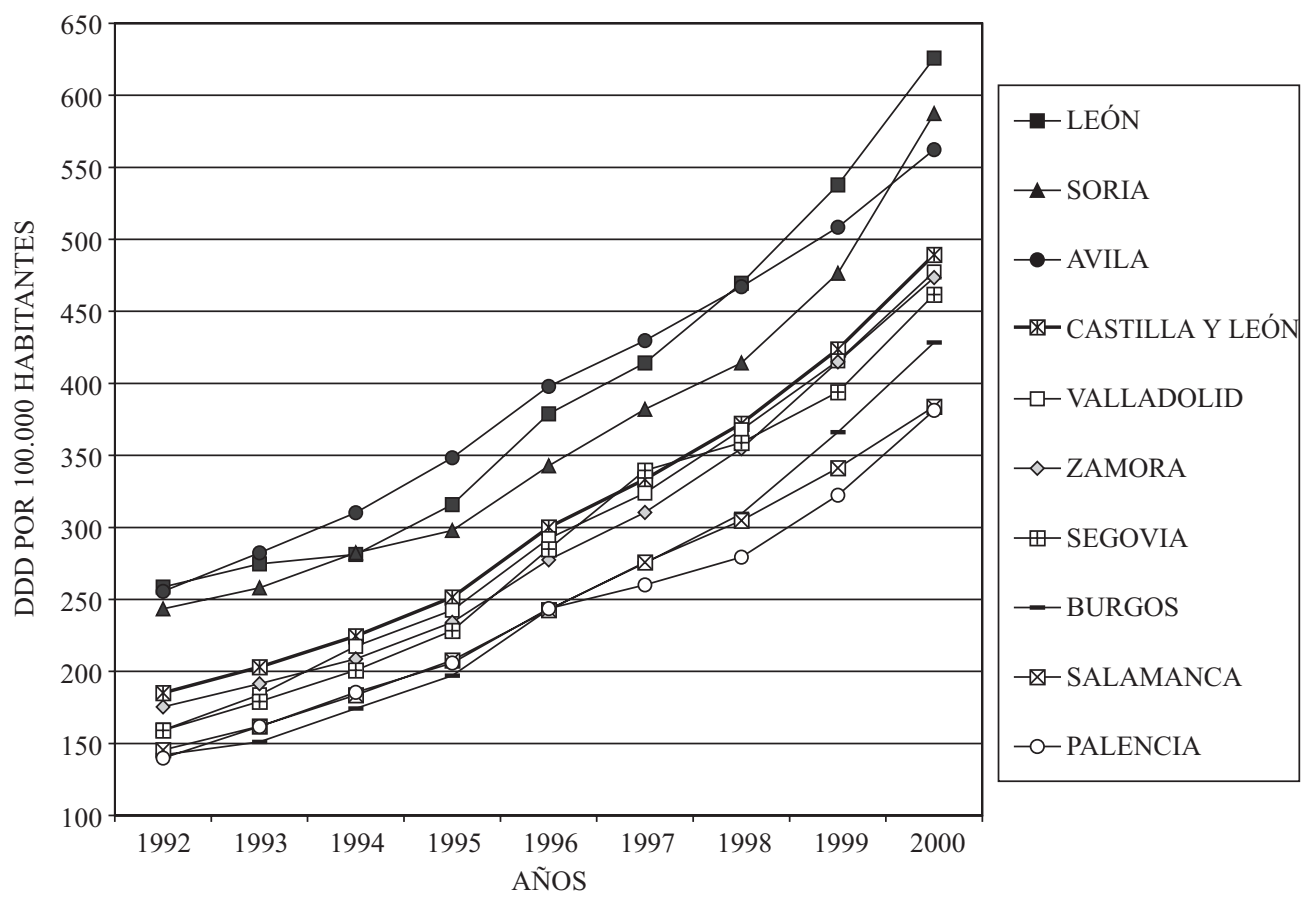

La evolución en el tiempo de la prevalencia en las diferentes provincias, se representa en la figura 1, observándose un incremento interanual en todas ellas. Las provincias con una mayor prevalencia durante todo el periodo son León (626 casos por 100.000 habitantes en el año 2000), seguido de Soria (587) y Ávila (562), estando ambas por encima del valor medio de la Comunidad. Las provincias con menor prevalencia son Palencia (381 casos por 100.000 habitantes en el año 2000), Salamanca (384) y Burgos (428).

\section{DISCUSIÓN}

Antes de analizar e interpretar los resultados obtenidos, hay que tener en cuenta una serie de limitaciones ya referenciadas en otras publicaciones ${ }^{11,15,17,20,21,22}$. En el estudio sólo se incluye el consumo extrahospita- lario de hormonas tiroideas a través de receta de la Seguridad Social, no conociendo la dispensación sin receta, ni los prescritos en el ámbito de otras entidades gestoras diferentes al Insalud o en el privado (ISFAS o MUFACE), por lo que el consumo real es necesariamente superior al obtenido. Sin embargo, consideramos que los envases dispensados sin receta representan un porcentaje muy pequeño y que dada la cobertura prácticamente universal del sistema público, es esperable que las diferencias entre lo hallado y lo real sean mínimas.

Se tienen datos de dispensación en oficina de farmacia, pero ello no quiere decir que los fármacos sean consumidos o administrados en su totalidad (incumplimiento terapéutico).

Las dosis diarias definidas empleadas pueden ser diferentes a las dosis realmente 
administradas, ya que la dosificación depende del grado de hipotiroidismo, la edad, grado de elevación de la TSH sérica, administración de otros fármacos, enfermedades asociadas o la indicación para la que se empleen y sin embargo se considera una sola DDD por fármaco.

Como población se ha utilizado las proyecciones y estimaciones intercensales de población de derecho publicadas por el Instituto Nacional de Estadística, contabilizándose colectivos como ISFAS, MUFACE, etc., a quienes no se les prescribe en receta de la Seguridad Social.

Al estimar la prevalencia, se calcula la referida a los casos que precisan tratamiento y son diagnosticados como tales en el sistema público, no incluyendo los cuadros subclíni$\cos$, los errores diagnósticos, los que afectan a la población no consultante, etc.

La prescripción de hormonas tiroideas en otras patologías como el bocio difuso o nodular, supone un pequeño porcentaje, lo que hace que su consumo este asociado prácticamente al tratamiento del hipotiroidismo.

Asumimos que todas estas limitaciones podrían dar lugar a una infraestimación de la prevalencia del hipotiroidismo, pero no invalidan los resultados, al ser representativos de la población y válidos para la inferencia epidemiológica, ya que se han obtenido de la misma fuente, de un solo grupo terapéutico, por periodos anuales y con el objetivo de observar la evolución de la prevalencia estimada en un periodo de tiempo ${ }^{11}$.

Hechas estas consideraciones, a través de las dosis diarias definidas por 100.000 habitantes aplicadas a la prescripción de hormonas tiroideas, estimamos que la prevalencia del hipotiroidismo en Castilla y León es en 1992 de 185 casos por 100.000 habitantes con un intervalo de confianza al $95 \%$ entre 180-190 y en el año 2000 de 489 casos por 100.000 con un intervalo de confianza al $95 \%$ entre $481-498$, lo que determina un incremento de la prevalencia de esta enferme- dad en la Comunidad, que pasa en términos absolutos de tener 4.693 enfermos a tener 12.144. Este incremento se observa en todas las provincias durante el periodo estudiado en distinto porcentaje, no teniendo por qué estar directamente relacionado con un aumento en la incidencia de la enfermedad, sino con otras variables, entre las que se encuentra el aumento de la población protegi$\mathrm{da}$, la progresiva mejora de la asistencia primaria, la mayor preparación de los profesionales médicos, el acceso más fácil a los servicios especializados y a los métodos de diagnóstico, así como al mejor seguimiento coordinado primaria-especializada ${ }^{23}$.

La naturaleza de los datos utilizados nos impide realizar estimaciones según el sexo y la edad, lo que dificulta la comparación con otros estudios ${ }^{4,5,8,9,10}$. La prevalencia estimada en Castilla y León en el año 2000 es de un $0,489 \%$, inferior a la obtenida por Ibarz et $\mathrm{l}^{5}$ en Lérida en 1994-95 que fue de 3,48 \pm $1,7 \%$. La diferencia de nuestros resultados con los de otros estudios pueden ser debidas a la distinta estructura demográfica y social, situación geográfica, variaciones en las metodologías empleadas, diferentes criterios diagnósticos y de inicio de tratamiento, etc.

En el estudio de Barbeira et $\mathrm{al}^{21}$ (1981-1982) con metodología similar, encontramos coincidencia en cuanto a que son León, Avila y Soria las provincias de la Comunidad con mayor prevalencia de hipotiroidismo y Salamanca y Palencia las de menor. Sin embargo, los valores de prevalencia encontrados en el año 1982 fueron inferiores a 35,6 por 100.000 habitantes para $\mathrm{Pa}$ lencia y Salamanca, mientras que en nuestro estudio son de 140 y 145 respectivamente en el año 1992; así mismo, la prevalencia es mayor a 52,4 para León, Ávila y Soria en 1982, mientras que en 1992 los valores encontrados por nosotros son de 259, 256 y 243 respectivamente.

Al no existir estudios en otras áreas geográficas de España, con metodología similar al nuestro y realizados en el mismo periodo, 
no podemos comparar nuestros resultados y establecer si el nivel de prevalencia encontrado en Castilla y León es alto o bajo con respecto a otras zonas.

Las diferencias observadas entre las provincias de la Comunidad pueden ser debidas a factores de riesgo de exposición diferente (ingesta de yodo), distinto uso de los servicios médicos, diferencias en la pirámide de población, aumento de la esperanza de vida u otras variables.

De lo anteriormente expuesto concluimos que los estudios de utilización de medicamentos constituyen un importante medio para la evaluación de la prescripción farmacológica, el conocimiento de las enfermedades y la generación de hipótesis, además de como marcadores simples y eficientes para estimar la prevalencia de ciertas enfermedades ${ }^{17}$ como el hipotiroidismo, ya que dada la especificidad del tratamiento se puede establecer una relación entre el consumo de hormonas tiroideas y la prevalencia de la enfermedad en la Comunidad de Castilla y León, así como la evolución de dicha prevalencia desde el año 1992 al 2000, aproximándonos a la situación actual de la prevalencia de hipotirodismo en esta Comunidad. A la vista de los resultados se observa un incremento en la prevalencia del hipotiroidismo en todas las provincias de la Comunidad.

Dado que la frecuencia del hipotiroidismo aumenta en zonas con déficit de yodo y, sin embargo, no se dispone de trabajos publicados en los que se dé una visión del problema en Castilla y León que nos permita relacionar el bocio endémico con nuestros resultados, sería deseable la realización de estudios que permitan conocer la situación de esta enfermedad en la Comunidad. Sugerimos la realización de estudios sobre la utilización de hormonas tiroideas, con la misma metodología y criterios de evaluación en otras provincias y comunidades, con el fin de conocer la prevalencia del hipotiroidismo y así comparar y contrastar nuestros resultados. Consideramos necesaria la realización de estudios con otra metodología que nos permitan conocer si factores como edad, sexo, lugar de residencia, etc., influyen en la prevalencia del hipotiroidismo en la Comunidad de Castilla y León, así como si la incidencia de la misma está aumentando.

\section{AGRADECIMIENTOS}

A la Subdirección General de Asistencia y Prestación Farmacéutica del Ministerio de Sanidad y Consumo por la información facilitada y a Don Tomás Martín González (Especialista en Endocrinología. Hospital Virgen de la Concha, Zamora) por sus sugerencias.

\section{BIBLIOGRAFÍA}

1. Cano Pérez JF, Bundó Vidiella M. Disfunción tiroidea. Alteraciones del calcio. En: Martín Zurro A, Cano Pérez JF. Atención primaria: conceptos, organización y práctica clínica. 4. ${ }^{\mathrm{a}} \mathrm{ed}$. Barcelona: Harcourt Brace de España; 1999. p. 1372-1407.

2. Olloqui Mundet J, Ochoa Prieto J, de Castro Pelegrín J. Tratamiento de las alteraciones de la función tiroidea en atención primaria (I): hipotiroidismo. Formación Médica Continuada en Atención Primaria 1996; 3: 325-330.

3. Canaris GJ, Manowitz NR, Mayor G, Ridgway EC. The Colorado thyroid disease prevalence study. Arch Intern Med 2000;160(4): 526-34.

4. Bjoro T, Holmen J, Kruger O, Midthjell K, Hunstad K, Schreiner T et al. Prevalence of thyroid disease, thyroid dysfunction and thyroid peroxidase antibodies in a large, unselected population. The Health Study of Nord-Trondelag (HUNT). Eur J Endocrinol 2000; 143(5): 639-47.

5. Ibarz Excuer M, Serra i Majem L, Gascó Eguiluz E, Serna Arnáiz MC, Vázquez Torget A, Peremiquel Lluch M. Prevalencia de trastorno de la función tiroidea en la provincia de Lleida. Aten Primaria 1999; 24: 475- 479.

6. Vila Ballester LI. Caracaterístiques clíniques i epidemiològiques del goll en la població de la Cerdanya. [Tesis doctoral]. Universitat Autònoma de Barcelona; 1993.

7. Tunbridge W, Evered D, Hall R, Appleton D, Brewis M, Clark F et al. The spectrum of thyroid 
disease in a community; the Whickham survey. Clin Endocrinol 1977; 7: 481-493.

8. Bagchy N, Brown T, Parish F. Thyroid dysfunction in adults over age 55 years. A study in urban us community. Arch Intern Med 1990: 150: 785-787.

9. Sawin CT Castelli WP, Hershman JM, McNamara $\mathrm{P}, \mathrm{Bacharach} \mathrm{P}$. The aging thyroid: thyroid deficiency in the Framingham Study. Arch Intern Med. 1985; 145: 1386-1388.

10. Hunter I, Greene SA, MacDonald TM, Morris AD. Prevalence and aetiology of hypothyroidism in the young. Arch Dis Child 2000; 83(3): 207-10.

11. Artazcoz Sanz MT, Viñas Rueda JJ. Estimación de la prevalencia de la enfermedad de Parkinson en Navarra. Estudio farmacoepidemiológico del consumo de antiparkinsonianos. Rev Esp Salud Pública 1995; 69: 479-485.

12. Memoria 1999. Dirección Territorial de Insalud de Castilla y León; 2000.

13. García Iñesta A. Estudios de utilización de medicamentos en España y análisis de la situación farmacoterapéutica. En: Instituto Nacional de la salud, editor. Estudios de utilización de medicamentos. Madrid: Insalud; 1992. p. 15-48.

14. Nomenclator Digitalis. Madrid: Insalud; 2001

15. Capellà D, Laporte JR. Métodos aplicados en estudios descriptivos de utilización de medicamentos. En: Laporte JR, Tognoni G, editores. Principios de epidemiología del medicamento. 4. ${ }^{\mathrm{a}} \mathrm{Ed}$ Barcelona: Masson-Salvat Medicina; 1993. p. 67-93.
16. García Ramos R. Introducción a la metodología de los estudios de utilización de medicamentos. En: Matos L, editor. Farmacoepidemiología. Santiago de Compostela: Xunta de Galicia; 1995. p. 205-221.

17. Criado-Álvarez JJ, Domper Tornil JA, de la Rosa Rodríguez G. Estimación de la prevalencia de trastornos bipolares tipo I en España a través de consumo de carbonato de litio (1996-1998). Rev Esp Salud Pública 2000; 74: 131-8.

18. Sartor F, Walckiers D. Estimate of disease prevalence using drug consumption data. Am J Epidemiol 1995; 141: 782-787.

19. Proyecciones de la población de España calculadas a partir del Censo de 1991. Instituto Nacional de Estadística (INE). Disponible en: URL: http://www.ine.es

20. Llop C, Ribas J, Rovira E. Estimación de la prevalencia de depresión a partir de la dosis diaria definida de antidepresivos. Med Clin (Barc) 1998; 110: 557-8.

21. Barbeira JM, García Iñesta A, Arias González A Consumo de hormonas tiroideas como indicador de enfermedades tiroideas. Inf Ter Segur Soc 1983; 7: 232-241.

22. Figueiras A, Caamaño F, Gestal Otero JJ. Metodología de los estudios de utilización de medicamentos en Atención Primaria. Gac Sanitaria 2000; 14 Supl 3: 7-19.

23. Álvarez Nido R, Pérez Calpena C, Alonso Roca $\mathrm{R}$, Monereo Mejías S. Papel del médico de atención primaria en el diagnóstico y seguimiento de la patología tiroidea. Aten Primaria 1994; 14: 877-879. 